\title{
On the Probability of Facing Fault Patterns: A Performance and Comparison Measure of Network Fault-Tolerance
}

\author{
Farshad Safaei ${ }^{1}$, Ahmad Khonsari ${ }^{3,2}$, and Reza Moraveji ${ }^{1,2}$ \\ ${ }^{1}$ Dept. of ECE, Shahid Beheshti Univ., Tehran, Iran \\ ${ }^{2}$ IPM School of Computer Science, Tehran, Iran \\ ${ }^{3}$ Dept. of ECE, Univ. of Tehran, Tehran, Iran \\ f_safaei@sbu.ac.ir, \{ak,moraveji_r\}@ipm.ir
}

\begin{abstract}
An important issue in the design and deployment of interconnection networks is the issue of network fault-tolerance for various types of failures. In designing parallel processing using torus as the underlying interconnection topology as well as in designing real applications on such processors, the estimates of the network reliability and fault-tolerance are important in choosing the routing algorithms and predicting their performance in the presence of faulty nodes. Under node-failure model, the faulty nodes may coalesce into fault patterns, which classified into two major categories, i.e., convex (l-shaped, $\square$-shaped) and concave (L-shaped, T-shaped, +-shaped, $\mathrm{H}$-shaped, U-shaped) regions. In this correspondence, we propose the first solution for computing the probability of message facing the fault patterns in tori both for convex and concave regions that is verified using simulation experiments. Our approach works for any number of faults as long as the network remains connected. We use these models to measure the network faulttolerance that can be achieved by adaptive routings, and to assess the impact of various fault patterns on the performance of such networks.
\end{abstract}

\section{Introduction}

Communication in faulty networks is a classical field in network theory. In practice, one cannot expect nodes or communication links to work without complications. Software or hardware faults may cause nodes or links to go down. To be able to adapt with faults without serious degradation of the service, networks and routing protocols have to be set up so that they are fault-tolerant. Several recent studies address faulttolerance in a diverse range of systems and applications [1-12]. Almost all of the performance evaluation studies for functionality of these systems, however, have made use solely of simulation experiments. The limitations of simulation-based studies are that they are highly time-consuming and expensive. Effective analytical models are necessary for predicting the behavior of large networks to help weigh the cost-performance trade-offs of various adaptive routing algorithms. In this correspondence, we focus specifically on the impact of the fault patterns that permits analytical model to predict the probability of facing fault patterns experienced by a 
message when an adaptive routing scheme is used. To the best of our knowledge, no study has been so far reported in the literature for calculating the probability of message facing the fault patterns to examine the relative performance merits of adaptive fault-tolerant routing algorithms. In this paper, we investigate the characteristics of fault patterns which are suitable for modeling faults in interconnections networks, particularly in torus topology. Our approach employs the theoretical results of algebra, and combinatorics to calculate the probability of occurrences of facing the fault patterns in a 2-D torus. Deriving expressions for characterizing fault patterns play a critical role in studying the performance of faulty networks by means of mathematical analysis.

The rest of the correspondence is organized as follows. In Section 2, we describe the basic properties of the torus topology as well as the fault-tolerance in networks. In Section 3, we derive an analytical model for calculating the probability of massage facing the fault patterns. In Section 4, the analytical results and comparison with simulation experiments are presented. Finally, Section 5 draws conclusions.

\section{Terminologies}

This section starts with a discussion of the torus structure and then provides a short summary of fault-tolerance in interconnection networks. Some of these definitions are reiterated from previous works $[6,7,10-12]$, for the sake of completeness.

\subsection{The Torus Topology}

The torus has been popular interconnection network topology in contemporary systems [6] due to their desirable properties, such as ease of implementation and ability to exploit communication locality to reduce message latency [12]. In addition, torus is a regular (i.e., all nodes have the same degree) and edge-symmetric network, which improves load balancing across the channels [13].

Definition 1 [13]: An $R \times C$ 2-D torus network, denoted by $T_{R \times C}$. Each node $\left(x_{1}, y_{1}\right)$ is connected to its four neighbors $\left(x_{1} \pm 1 \bmod R, y_{1}\right)$ and $\left(x_{1}, y_{1} \pm 1 \bmod C\right)$. Therefore, the total number of channels in torus $T_{R \times C}$ is $E=2 \times R \times C$.

\subsection{Network Fault-Tolerance}

The growth of parallel applications on multiprocessors system-on-chip (Mp-SoCs), multicomputers, cluster computers, and peer-to-peer systems motivates interest in parallel computer networks. The construction of such networks connecting a large population of processing units and components (such as routers, channels and connectors) poses several challenges. First, selecting the right routing algorithms should reflect the full potential of the underlying network topology. Second, connectivity among active nodes of the interconnection network should be maintained, even in the presence of high failure rates or when a large portion of nodes is not active. To seek solutions to these issues, adaptive fault-tolerant routing algorithms have been frequently suggested as a means of providing continuous operations in the presence of one or more 
failures by allowing the graceful system degradation. In designing a fault-tolerant routing algorithm, a suitable fault model is one of the most important issues [7-10]. The fault model can reflect fault situations in a real system. Rectangular fault modes (also known as block faults) are the most common approach to model faulty nodes and to facilitate routing in 2-D tori [9]. However, rectangular fault regions sacrifice many nonfaulty nodes and hence its resources are wasted. In order to reduce non-faulty nodes in rectangular fault regions, many studies have addressed the concept of fault patterns with different shapes $[7-10,12]$ may form convex or concave regions. A convex region is defined as a region $\varphi$ in which a line segment connecting any two points in $\varphi$ lies entirely within $\varphi$. If we change the "line segment" in the standard convex region defini tion to "horizontal or vertical line segment", the resulted region is called rectilinear convex segments $[7,10]$. Any region that is not convex is a concave region. Examples of convex regions are I-shape, $\square$-shape and concave regions are L-shape, U-shape, Tshape, H-shape, +-shape. The detailed mathematical expressions for characterization of the most common concave and convex fault regions in torus and mesh networks have been reported in [14]. For a comprehensive survey of the important issues of the faulttolerant systems and networks, the reader is referred to the articles in [12-15].

\section{Mathematical Analysis}

This section starts with the description of the assumptions used in construction of the analytical models. The derivation and implementation procedure of the mathematical models are then presented. After that, the proposed models are validated through simulation experiments.

\subsection{Assumptions}

The analytical models are based on common assumptions that have been widely accepted in the literature $[6,7,9,11-15]$.

i. Messages are uniformly directed to other network nodes.

ii. Messages are routed adaptively through the network. Further, a message is assumed to always follow one of the available shortest paths in the absence of faults.

iii. The probabilities of node failure in the network are equiprobable and independent of each other. Moreover, fault patterns are static [6, 7, 9-15] and do not disconnect the network.

iv. Nodes are more complex than links and thus have higher failure rates $[7,12,14]$. So, we assume only node failures.

\subsection{Calculating the Probability of Message Facing Faulty Patterns}

Consider an $R \times C$ torus network in which there are some faulty nodes have formed one of the fault patterns so that faulty nodes do not disconnect the network. We call such network a connected $R \times C$ torus with the $X-$ shape fault pattern. 
In this section, our goal is to calculate the probability of a message facing the existing fault pattern in the connected $R \times C$ torus network in the presence of the $X$ - shape fault pattern.

Remark: A path facing the fault-pattern means that there exists one or more points from the set of points reside on the given path.

In the torus network, the position of the fault patterns does not play an important role. Since, by changing the coordinate we can transfer these patterns to any other location in the network without changing the location of the nodes respect to each other. Therefore, we can obtain the exact shape of that by knowing the type of the fault pattern and some characteristics. We denote the set of these characteristics in the $X$ - shape fault pattern by $S_{X}$. In the rectangular fault pattern, the determining characteristics of such regions are its height and width that are indicated by $l$ and $h$, respectively. Thus, $S_{\square}: l, h$.

For instance, in the I-shape fault pattern, the determining characteristics of the shape of this line segment are its vertical height or its horizontal width.

\begin{tabular}{|c|c|}
\hline$S_{\mid}: 1, h$ & Vertical line segment \\
\hline$S_{\mid}: l, 1$ & Horizontal line segment \\
\hline
\end{tabular}

Fig. 1 depicts some of the commonest fault patterns together with the precise determining characteristics of them. In the fault patterns with different horizontal and vertical determining characteristics, it is possible to alter the horizontal case to vertical case by changing the role of $R$ and $C$ in the torus network. Therefore, for all the proposed fault patterns, the determining characteristics in the vertical case are adequate.

In case that the set of fault points are not in any of the mentioned above fault patterns, we should know the coordinate of the new fault pattern points or define new characteristics according to its shape. Here, we investigate those fault patterns about which we know the determining characteristics of their exact shape in addition to having information of their general shape. The set of $X-$ shape fault pattern points with $S_{X}$ characteristics is demonstrated by $F\left(X ; S_{X}\right)$ and the probability of a path confronting it is illustrated by $P\left(X ; S_{X}\right)$. In order to calculate the parameter $P\left(X ; S_{X}\right)$, we should enumerate the number of all existing paths facing the $X$ - shape fault pattern and divide them by the number of all existing paths in the connected $R \times C$ torus network. This probability is expressed formally as

$$
P_{\text {hit }} \triangleq \frac{\text { The number of minimal paths crossing the fault region }}{\text { The number of all minimal paths existing in the network }}
$$

The following theorem provides the total number of paths with minimal length in the network.

Theorem 1: In a connected $R \times C$ torus network with the $X-$ shape fault region, the number of all existing paths between any pair of non-faulty nodes is denoted by 


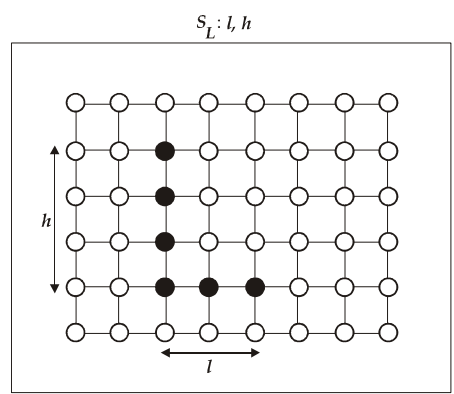

(a)

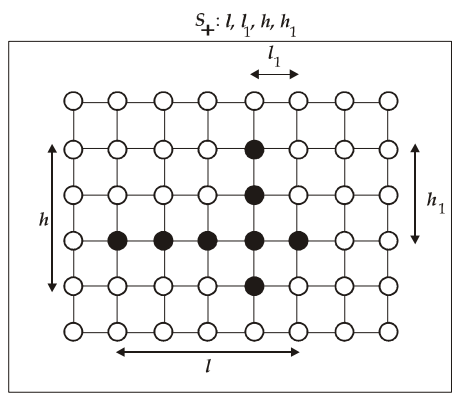

(c)

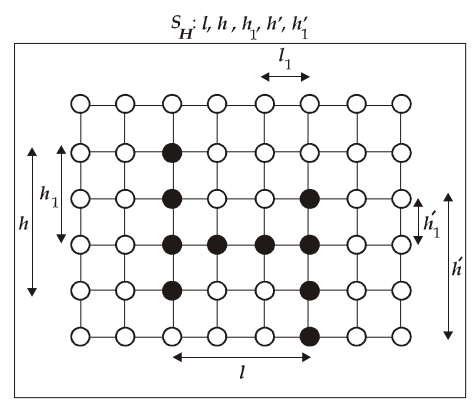

(e)

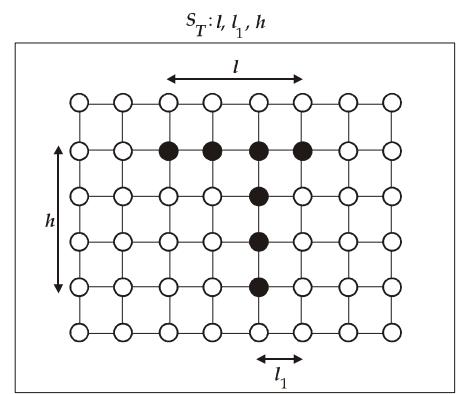

(b)

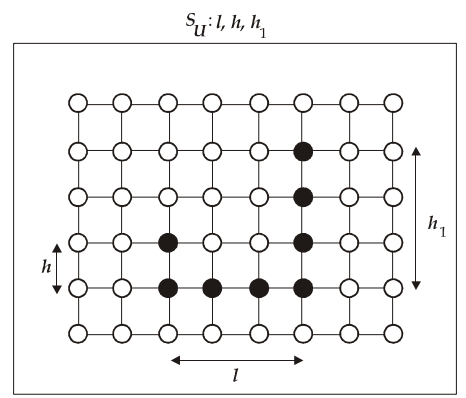

(d)

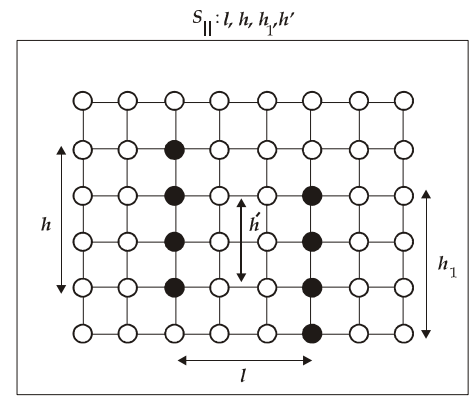

(f)

Fig. 1. Examples of fault patterns in a 2-D torus network

$$
\sum_{a, b \in v\left(T_{R \times C} \backslash F\left(X ; S_{X}\right)\right.} L T(a, b)
$$

where $F\left(X ; S_{X}\right)$ is the set of $X$ - shape faulty points with determining characteristics $S_{X}$.

Proof: Consider a connected $R \times C$ torus with the $X$-shape fault pattern. Consider two non-faulty points $a$ and $b$ in the mentioned above network. The number of paths crossing from $a$ to $b$ is given by

$$
L T(a, b)
$$


Thus, the number of all existing paths in the above network can be calculated as the aggregate of the total number of paths crossing between any two of non-faulty points in the network

$$
\sum_{a, b \in v\left(T_{R \times C}\right) \backslash F\left(X ; S_{X}\right)} L T(a, b)
$$

which completes the proof.

Example 1: Consider an $8 \times 7$ torus network in which the embedded T-shaped fault region has three determining characteristics $l=7, h=5$, and $h_{1}=3$ (see Fig. 2). We wish to route messages from point $a$ to point $b$. In this network, there is a minimal path from $a$ to $b$ as follows

$$
a=(6,3) \rightarrow(6,1) \rightarrow(6,7) \rightarrow(5,7) \rightarrow(4,7)=b
$$

Therefore, the set of first components of the existing nodes along this path is $\{5,6,4\}$ and also the set of second components of the existing nodes in this path is $\{3,2,1,7\}$. So, we get

$$
\begin{aligned}
M(a, b)=\{6,5,4\} \times\{3,2,1,7\}= & \{(6,3),(6,2),(6,1),(6,7),(5,3),(5,2), \\
& (5,1),(5,7),(4,3),(4,2),(4,1),(4,7)\}
\end{aligned}
$$

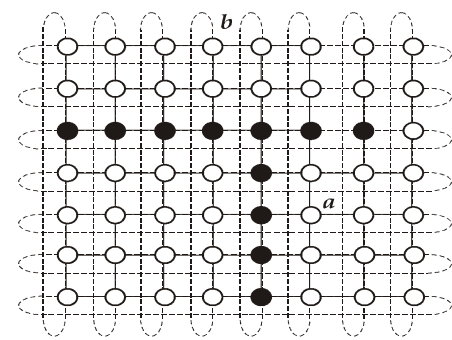

(a)

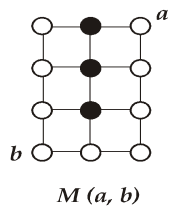

(b)

Fig. 2. (a) A torus network with two arbitrary points $a$ and $b$ in the presence of T-shaped fault pattern; (b) Demonstration of $M(a, b)$ mesh subnetwork.

Before we proceed to calculate Equation (1), we pause to give a few definitions; then we present and prove a theorem.

Definition 2: Let $a$ and $b$ be two non-faulty points of $v\left(T_{R \times C}\right)$. For any two arbitrary points $C_{i}=\left(x_{C_{i}}, y_{C_{i}}\right)$ and $C_{j}=\left(x_{C_{j}}, y_{C_{j}}\right)$ of $\mathcal{R}(M(a, b))$, the number of possible paths from $C_{j}$ to $C_{i}$ is indicated by $L M_{a, b}\left(C_{j}, C_{i}\right)$ so that the direction of each path in dimension $X(Y)$ is collinear with the direction of a path from a to $b$ in dimension $(X) Y$. Let us attempt to define $L M_{a, b}\left(C_{j}, C_{i}\right)$ by

$$
\left(\begin{array}{c}
\Theta_{x}^{a, b}\left(C_{j}, C_{i}\right)+\Theta_{y}^{a, b}\left(C_{j}, C_{i}\right) \\
\Theta_{x}^{a, b}\left(C_{j}, C_{i}\right)
\end{array}\right)
$$


in which $\Theta_{x}^{a, b}\left(C_{j}, C_{i}\right)$ is a function indicating the number of orientations along a path from $C_{j}$ to $C_{i}$ in dimension $X$ which are collinear with the orientations along a path from $a$ to $b$ and it's criterion is expressed as

$$
\Theta_{x}^{a, b}\left(C_{j}, C_{i}\right)=\left\{\begin{array}{cl}
\Delta_{x}\left(C_{j}, C_{i}\right) & \left(0 \geq x_{b}-x_{a} \geq-\left\lfloor\frac{R}{2}\right\rfloor \text { or } x_{b}-x_{a}>\left\lfloor\frac{R}{2}\right\rfloor\right) \text { and } \\
& \left(0 \geq x_{C_{i}}-x_{C_{j}} \geq-\left\lfloor\frac{R}{2}\right\rfloor \text { or } x_{C_{i}}-x_{C_{j}}>\left\lfloor\frac{R}{2}\right\rfloor\right) \\
\Delta_{x}\left(C_{j}, C_{i}\right) & \left(0 \leq x_{b}-x_{a} \leq\left\lfloor\frac{R}{2}\right\rfloor \text { or } x_{b}-x_{a}<-\left\lfloor\frac{R}{2}\right\rfloor\right) \text { and } \\
& \left(0 \leq x_{C_{i}}-x_{C_{j}} \leq\left\lfloor\frac{R}{2}\right\rfloor \text { or } x_{C_{i}}-x_{C_{j}}<-\left\lfloor\frac{R}{2}\right\rfloor\right) \\
-\Delta_{x}\left(C_{j}, C_{i}\right) & \text { otherwise }
\end{array}\right.
$$

Similarly, we can obtain the criterion of function $\Theta_{y}^{a, b}\left(C_{j}, C_{i}\right)$ by interchanging the roles of $X$ and $Y$ as

$$
\Theta_{y}^{a, b}\left(C_{j}, C_{i}\right)=\left\{\begin{array}{cl}
\Delta_{y}\left(C_{j}, C_{i}\right) & \left(0 \geq y_{b}-y_{a} \geq-\left\lfloor\frac{R}{2}\right\rfloor \text { or } y_{b}-y_{a}>\left\lfloor\frac{R}{2}\right\rfloor\right) \text { and } \\
& \left(0 \geq y_{C_{i}}-y_{C_{j}} \geq-\left\lfloor\frac{R}{2}\right\rfloor \text { or } y_{C_{i}}-y_{C_{j}}>\left\lfloor\frac{R}{2}\right\rfloor\right) \\
\Delta_{y}\left(C_{j}, C_{i}\right) & \left(0 \leq y_{b}-y_{a} \leq\left\lfloor\frac{R}{2}\right\rfloor \text { or } y_{b}-y_{a}<-\left\lfloor\frac{R}{2}\right\rfloor\right) \text { and } \\
& \left.\left(0 \leq y_{C_{i}}-y_{C_{j}} \leq\left\lfloor\frac{R}{2}\right\rfloor \text { or } y_{C_{i}}-y_{C_{j}}<-\mid \frac{R}{2}\right\rfloor\right) \\
-\Delta_{y}\left(C_{j}, C_{i}\right) & \text { otherwise }
\end{array}\right.
$$

Theorem 2: Given that $a$ and $b$ are two non-faulty points of a torus network and $\mathcal{R}(M(a, b))=\left\{C_{1}, C_{2}, \ldots, C_{k}\right\}$, the number of paths from a to $b$ that do not traverse the points $C_{1}, C_{2}, \ldots, C_{k}$ can be calculated as

$$
\operatorname{det}_{0 \leq i, j \leq k} d_{i j}(a, b)
$$

where

$$
\begin{array}{ll}
d_{0 j}(a, b)=L M_{a, b}\left(C_{j}, C_{k+1}\right) & j=0,1, \ldots, k \\
d_{i j}(a, b)=L M_{a, b}\left(C_{j}, C_{i}\right) & i=1,2, \ldots, k \quad j=0,1, \ldots, k
\end{array}
$$

Proof: The proof is quite involved and we omit it due to lack of space. The interested reader is referred to [16] for the proof.

Theorem 3: Let $T_{R \times C}$ be a connected $R \times C$ torus network with the $X-$ shape fault region having $S_{X}$ characteristics. The number of paths in $T_{R \times C}$ not facing the fault pattern is expressed as 


$$
\sum_{a, b \in v\left(T_{R \times C}\right) \backslash F\left(X ; S_{X}\right)} \operatorname{det}_{0 \leq i, j \leq C_{a, b}} d_{i j}(a, b)
$$

in which $C_{a, b}$ is the number of elements of $\mathcal{R}(M(a, b))$; that is

$$
\|\mathcal{R}(M(a, b))\|=C_{a, b}
$$

Proof: Consider two arbitrary points $a$ and $b$ from the set $v\left(T_{R \times C}\right) \backslash F\left(X ; S_{X}\right)$. According to Theorem 2, the number of minimal paths (from $a$ to $b$ ) not traversing the points $\mathcal{R}(M(a, b))$, equals $\operatorname{det}_{0 \leq i, j \leq C_{a, b}} d_{i j}(a, b)$. Therefore, the number of minimum paths from from $a$ to $b$ not crossing the $F\left(X ; S_{X}\right)$ points, will be equal to $\operatorname{det}_{0 \leq i, j \leq C_{a, b}} d_{i j}(a, b)$. So, the number of all existing paths in $T_{R \times C}$ not traversing the $F\left(X ; S_{X}\right)$ points is equal to aggregate of the total number of paths between any two non-faulty points in $T_{R \times C}$ not traversing the points of $F\left(X ; S_{X}\right)$. That is,

$$
\sum_{a, b \in v\left(T_{R \times C}\right) \backslash F\left(X ; S_{X}\right)} \operatorname{det}_{0 \leq i, j \leq C_{a, b}} d_{i j}(a, b)
$$

It follows from the preceding theorem, the probability that a path in $T_{R \times C}$ not facing the fault pattern, $P_{\text {miss }}$, is given by

$$
\frac{\sum_{a, b \in v\left(T_{R \times C}\right) \backslash F\left(X ; S_{X}\right)} \operatorname{det}_{0 \leq i, j \leq C_{a, b}} d_{i j}(a, b)}{\sum_{a, b \in v\left(T_{R \times C}\right) \backslash F\left(X ; S_{X}\right)} L T(a, b)}=\sum_{a, b \in v\left(T_{R \times C}\right) \backslash F\left(X ; S_{X}\right)} \frac{\operatorname{det}_{0 \leq i, j \leq C_{a, b}} d_{i j}(a, b)}{L T(a, b)}
$$

Therefore, it is trivial that

$$
P_{h i t}=1-P_{\text {miss }}=1-P\left(X ; S_{X}\right)=1-\sum_{a, b \in v\left(T_{R \times C}\right) \backslash F\left(X ; S_{X}\right)} \frac{\operatorname{det}_{0 \leq i, j \leq C_{a, b}} d_{i j}(a, b)}{L T(a, b)}
$$

\section{Experimental Results}

In the previous sections, we have derived mathematical expressions to calculate the probability of facing the fault patterns. These analytical expressions form the core of other fault patterns computation in other topologies and can be extensively generalized. An experimental approach is necessary to verify the analytical evaluation to which mathematical analysis led. A program has been developed which simulates the failure of nodes and the subsequent constructing of the corresponding fault patterns. The simulator generates faults in the network so that the resulting fault regions are convex or concave. It also checks that all nodes in the network are still connected using adaptive routing. The objective of the simulation is to measure the values of the probability of facing the fault patterns for different values of faulty nodes in the torus topology. For every run, the simulator creates the fault pattern and keeps statistics of the following data: 
- The number of minimal paths crossing the network.

- The number of minimal paths confronting the fault pattern.

- For each source - destination pair, the probability of facing the fault pattern is computed.

Table 1 reveals the results obtained from simulation experiments and the mathematical models in torus for different sizes of the network and various shapes of fault patterns.

Table 1. Experimental results of the probability of facing fault patterns in the torus with different fault patterns, and various sizes of the network which agrees with the analytical expressions

\begin{tabular}{|c|c|c|c|c|c|}
\hline & \multicolumn{5}{|c|}{ Torus Network $\left(T_{R \times C}\right)$} \\
\hline Fault patterns characteristics & $9 \times 13$ & $10 \times 10$ & $11 \times 9$ & $6 \times 7$ & $6 \times 5$ \\
\hline I-shape, $h=4$ & 0.173 & 0.165 & 0.147 & 0.214 & 0.194 \\
\hline I-shape, $l=3$ & 0.109 & 0.131 & 0.134 & 0.156 & 0.191 \\
\hline I-shape, $h=h_{1}=3, l=3, h^{\prime}=2($ case 1$)$ & 0.208 & 0.219 & 0.201 & 0.310 & 0.377 \\
\hline II-shape, $h=4, h_{1}=3, l=2, h^{\prime}=2($ case 2$)$ & 0.212 & 0.201 & 0.183 & 0.257 & 0.244 \\
\hline L-shape, $h=3, l=3$ & 0.165 & 0.182 & 0.170 & 0.229 & 0.263 \\
\hline L-shape, $h=4, l=5$ & 0.247 & 0.286 & 0.273 & 0.395 & 0.429 \\
\hline T-shape, $l=4, h=3, l_{1}=2$ & 0.178 & 0.201 & 0.194 & 0.261 & 0.305 \\
\hline T-shape, $l=5, h=4, l_{1}=4$ & 0.237 & 0.261 & 0.257 & 0.375 & 0.397 \\
\hline U-shape, $l=3, h=4, h_{1}=2$ & 0.207 & 0.219 & 0.202 & 0.296 & 0.353 \\
\hline$\square$-shape, $l=3, h=2$ & 0.133 & 0.150 & 0.147 & 0.170 & 0.195 \\
\hline H-shape, $l=3, h=4, h_{1}=3, h^{\prime}=3, h_{1}^{\prime}=2$ & 0.213 & 0.226 & 0.209 & 0.317 & 0.392 \\
\hline H-shape, $l=5, h=4, l_{1}=4, h_{1}=2$ & 0.216 & 0.243 & 0.239 & 0.339 & 0.346 \\
\hline
\end{tabular}

\section{Conclusions}

In recent years, efforts have been made to integrate performance and reliability of adaptive routing algorithms to overcome the drawback in the traditional evaluation methods for interconnection networks. For this purpose, a new performance metric of network reliability, probability of facing fault patterns, has been introduced. It is used to assess the performance-related reliability of such routing schemes in the presence of faulty patterns, which can be categorized to two major classes of convex (I-shape, $\square$-shape) and concave (L-shape, U-shape, +-shape, T-shape, and H-shape) regions. In this paper, we have attempted to derive mathematical expressions for calculating the probability of message facing fault patterns occur in adaptively-routed torus networks. Predicting the network measures, such as message latency and channel waiting times throughout a faulty network are applications of the results derived in this paper. Since, 
the mesh topology has become a popular interconnection architecture for constructing massively parallel computers; a more challenging extension of our work would be to propose mathematical expressions for fault patterns in the well-known mesh topologies.

\section{References}

1. Chakravorty, S., Kalé, L.V.: A Fault Tolerant Protocol for Massively Parallel Systems. In: Proceedings of the 16th International Symposium on Parallel and Distributed Processing (2004)

2. Al-Karaki, J.N.: Performance Analysis of Repairable Cluster of Workstations. In: Proceedings of the 16th International Symposium on Parallel and Distributed Processing (2004)

3. Karimou, D., Myoupo, J.F.: A Fault-Tolerant Permutation Routing Algorithm in Mobile Ad-Hoc Networks. In: Lorenz, P., Dini, P. (eds.) ICN 2005. LNCS, vol. 3421, pp. 107 115. Springer, Heidelberg (2005)

4. Gupta, G., Younis, M.: Fault-tolerant clustering of wireless sensor networks. In: IEEE Conf. on Wireless Communications and Networking, pp. 1579-1584 (2003)

5. Pande, P.P., et al.: Performance Evaluation and Design Trade-Offs for Network-on-Chip Interconnect Architectures. IEEE Trans. Computers 54(8), 1025-1040 (2005)

6. Dao, B.V., Duato, J., Yalamanchili, S.: Dynamically configurable message flow control for fault-tolerant routing. IEEE Transactions on Parallel and Distributed Systems 10(1), 7-22 (1999)

7. Suh, Y.J., et al.: Software-based rerouting for fault-tolerant pipelined communication. IEEE Trans. on Parallel and Distributed Systems 11(3), 193-211 (2000)

8. Chen, C.L., Chiu, G.M.: A Fault-tolerant routing scheme for meshes with nonconvex faults. IEEE Trans. on Parallel and Distributed Systems 12(5), 467-475 (2001)

9. Shih, J.-D.: Fault-tolerant wormhole routing in torus networks with overlapped block faults. IEE Proc.-Comput. Digit. Tech. 150(1), 29-37 (2003)

10. Wu, J., Jiang, Z.: On Constructing the Minimum Orthogonal Convex Polygon in 2-D Faulty Meshes, IPDPS (2004)

11. Theiss, I.: Modularity, Routing and Fault Tolerance in Interconnection Networks, PhD thesis, Faculty of Mathematics and Natural Sciences, University of Oslo (2004)

12. Gómez, M.E., et al.: A Routing Methodology for Achieving Fault Tolerance in Direct Networks. IEEE Transactions on Computers 55(4), 400-415 (2006)

13. Duato, J., Yalamanchili, S., Ni, L.M.: Interconnection networks: An engineering approach. Morgan Kaufmann Publishers, San Francisco (2003)

14. Hoseiny Farahabady, M., Safaei, F., Khonsari, A., Fathy, M.: Characterization of Spatial Fault Patterns in Interconnection Networks. Journal of Parallel Computing 32(11-12), 886$901(2006)$

15. Xu, J.: Topological structure and analysis of interconnection networks. Kluwer Academic Publishers, Dordrecht (2001)

16. Safaei, F., Fathy, M., Khonsari, A., Gilak, M., Ould-Khaoua, M.: A New Performance Measure for Characterizing Fault-Rings in Interconnection Networks. Journal of Information Sciences (submitted, 2007) 